\title{
Reactions with Visnaginone: Synthesis, Cyclisation and Microbial Evaluation of Some Visnaginone Thiosemicarbazone Derivatives
}

\author{
Sadek Elsayed Abdou \\ Department of Chemistry, Faculty of Science, Cairo University, Giza, Egypt
}

Salah Mohamed El-Qusy

Department of Chemistry, Faculty of Science, Minofia University, Shebein Elkoum, Egypt

Sami Selim Ghabrial

National Organization for Drug Control and Research (NODCAR), Dokki, Giza, Egypt

Mahmoud Ibrahim Haggag (Corresponding author)

Macro Group pharmaceutical Co. Badr City, Cairo, Egypt

Tel: 202-01-0545-8068, 202-01-0214-4710, 202-01-4584-5200Ｅ-mail: hagag2610@yahoo.com

Received: August 11, 2011

Accepted: August 29, 2011

doi:10.5539/mas.v5n5p140

\begin{abstract}
Several new visnaginone ethers were prepared and their thiosemicarbazone derivatives are synthesized. The behavior of the thiosemicarbazones in methanolic sodium methoxide and hydrochloric acid is discussed. Structures were established on the bases of elemental and spectral data studies. Some of the thiosemicarbazones were tested for their antimicrobial activity. The structures of the synthesized derivatives (5a-g,6a-g) were confirmed by means of IR, 1H NMR, MS and elemental analyses. The synthesized derivatives (5a-g,6a-g) wear subjected to the Microbiology Division, Microanalytical Center, Cairo University, (5a-g,6a-g) showed a variable degree of antimicrobial activity.
\end{abstract}

Keywords: Visnaginone, Thiosemicarbazones, 1,2,4-Triazolylbenzofurans, Benzopyrans

\section{Introduction}

Visnagine and its derivatives are long known to possess diverse biological activities (McClure et al., 1975, Bruneton, 1995,Hishmat et al., 2002). Various thiosemicarbazides (Achary and Rao, 1992). and their cyclised derivatives such as triazoles (Maria et al.,1980), Oxadiazoles (Clarcke and Roberttson,1949 ,Geissmann and Halshall,1951), thiadizolinones (Rene and Christophe, 1977,Simonis and Rosenberg,1914), and thiadiazoles are also associated with a broad spectrum of biological activities that include analgesic (Clarcke and Roberttson, 1949,Rajanna et al.,1996), antiproteolytic (Chaudhary et al.,1978), anti-inflammatory (Middleton et al.,1994), muscle relaxant (Geissmann and Halshall,1951), antifungal and antibacterial activities (Harborne and Williams,2000). It was thus of value to synthesis a number of new derivatives for biological activity screening together with a study of their behavior in buffered and acidic media.

Thus, it has been found that visnaginone 2, preparedly hydrolysis of Visnagine 1 as described in literature (Anteunis et al., 1972). reacted with methyl iodide to give a reaction product of molecular formula $\mathrm{C}_{12} \mathrm{H}_{12} \mathrm{O}_{4}$ corresponding to equimolecular addition of 2 to the reagent followed by the loss of one molecule of hydrogen iodide.

\section{Results and Discussion:}

\subsection{Chemistry}

The prepared ethers were reacted with thiosemicarbazide (4) and the behavior of the resultant reaction products towards buffered and acidic media was also investigated. 
Thus, it has been found that (3a) reacted with (4) in boiling methanolic sodium methoxide solution in the presence of sodium acetate as a buffering agent to give a reaction product of molecular formula $\mathrm{C}_{13} \mathrm{H}_{15} \mathrm{~N}_{3} \mathrm{O}_{3} \mathrm{~S}$. This formula corresponded to equimolecular addition of (3a) to (4) followed by loss of the elements of water. The band related to the presence of the acetyl-CO group was entirely absent in the IR spectrum of the reaction product and instead the bands of new $\mathrm{NH}$ and $\mathrm{NH}_{2}$ groups were detected. Moreover, the ${ }^{1} \mathrm{H}-\mathrm{NMR}$ of the reaction product revealed the presence of side chain- $\mathrm{CH}\left(\mathrm{s}, 0.9 \delta \mathrm{ppm}\right.$ ), two $\mathrm{OCH}_{3}$ groups (as two singlet's at 3.7 and $3.75 \delta \mathrm{ppm}$ ) in addition to the aromatic- $\mathrm{CH}(\mathrm{s}, 6.5 \delta \mathrm{ppm})$, the furan $\mathrm{H}-3(\mathrm{~d}, 6,7)$, the furan $\mathrm{H}-2(\mathrm{~d}, 7.4)$, the $\mathrm{NH}(\mathrm{s}, \mathrm{br}, 7.0 \delta \mathrm{ppm})$ and the $\mathrm{NH}_{2}$ (s, br, $2 \delta \mathrm{ppm}$ ) groups. (cf. Experimental Part). (Scheme II). Based on the above data the reaction product was formulated as 1-[(4,6-dimethoxybenzo-furan-5-yl)ethylidene] thiosemicarbazide (5a).

On performing the above reaction in methanolic $\mathrm{HCl}$ a product of molecular formula $\mathrm{C}_{13} \mathrm{H}_{15} \mathrm{~N}_{3} \mathrm{O}_{3} \mathrm{~S}$ was obtained. This is exactly the same formula of (5a). A much depression in the melting point was observed on admixture of a sample of the two compounds. It was thought at first that it is a matter of isomserism analogous to that previously reported for semicarbazones (Huttrer and Dale,1951,Martinez and Miranda,1981,Heilbron et al.,1923, Spath and Gruber,1938,Spath and Gruber,1941). A type of syn- and anti-forms seemed reasonable. Interconversion of isomers is relatively easy and can be achieved smoothly by heat or by acids. However, careful examination of the ${ }^{1} \mathrm{H}-\mathrm{NMR}$ spectra of (5a) and the reaction product showed the absence of the signal of the $\mathrm{NH}_{2}$ group at about $5.7 \delta$ ppm. Argument against possible syn-anti isomerism are (a)- the close relationship between the electronic spectra of both isomers and (b)- the slow inter conversion between both isomers during running of the ${ }^{1} \mathrm{H}$-NMR spectra. The observed isomerism can be explained in terms of a ring closure structure supported by the results of ${ }^{1} \mathrm{H}-\mathrm{NMR}$ data. Consequently the reaction product could be formulated as 5-(4,6-dimethoxybenzo-furan-5-yl)-5-methyl-1,2,4-triazoline-3-thione (6a).

A further support for the structure of (6a) was achieved by synthesis of the compound via another route by boiling a solution of (5a) in methanolic $\mathrm{HCl}$. Compound(6a) prepared via this route was found completely identical in all aspects (m.p., mixed m.p., analysis and spectral data) as (6a) prepared as reported previously (cf. Experimental Part). (Scheme II).

Similarly, each of (3b-g) reacted with (4) to afford 1-[(4-methoxy,6-ethoxybenzofuran-5-yl) ethylidene]thiosemicarbazide (5b), 1-[(4-methoxy-6-n-propoxybenzofuran-5-yl)ethylidene]thiosemi carbazide (5c), 1-[(4-methoxy-(6-isopropoxybenzofuran-5-yl) ethylidene]thiosemicarbazide (5d), 1-[(4-methoxy-6-n-butyloxybenzofuran-5-yl)ethylidene]thiosemicarbazide(5e), 1-[(4-methoxy-6-allyloxy benzofuran-5-yl)ethylidene]thiosemicarbazide(5f),1-[(4-methoxy-6-benzyloxybenzofuran-5-yl) ethylidene] thiosemicarbazide $(5 \mathrm{~g})$ respectively.

On the other hand, the reaction of each of (3b-g) with (4) in ethanolic $\mathrm{HCl}$ resulted in the formation of 5(4-methoxy-6-ethoxybenzofuran-5-yl)-5-methyl-1,2,4-triazoline-3-thione (6b), 5-(4-methoxy-6-n-propyl oxybenzyloxybenzofuran-5-yl)-5-methyl-1,2,4-triazoline-3-thione (6c), 5-(4-methoxy-6-iso-propyloxy benzyloxybenzofuran-5-yl)-5-methyl-1,2,4-triazoline-3-thione (6d), 5-(4-methoxy-6-n-butyloxybenzyl oxybenzofuran-5-yl)-5-methyl-1,2,4-triazoline-3-thione (6e), 5-(4-methoxy-6-n-allyloxybenzyloxy benzofuran-5-yl)-5-methyl-1,2,4-triazoline-3-thione

And 5-(4-methoxy-6-benzyloxybenzyloxybenzo-furan-5-yl)-5-methyl-1,2,4-triazoline-3-thione (6g) respectively. The structure of (6b-g) was also established on the basis of elemental and spectroscopic data studies (cf. Experimental Part). (Scheme II).

On the other hand, compounds (5a-f) were also obtained via another route. Visnaginone (2) reacted with thiosemicarbazide hydrochloride in boiling methanol in the presence of anhydrous sodium acetate to give visnaginone thiosemicarbazone (7) whose structure was based on elemental and spectral data (cf. Experimental Part). (Scheme II). Compound (7) could, in turn, be reacted with the appropriate aryl or aralkyl halides in the usual manner followed by basic hydrolysis (to hydrolyses any $\mathrm{N}$-alkyl derivatives which may be formed) to furnish the corresponding (5a-f) with m.ps. and mixed m.ps, analytical and spectral data as those previously prepared via the first route. (Scheme II). Finally, A start compound that undergoes hydrolysis which react with aralkyl halide to form a novel compounds (3a-g). This compounds react with thiosemicarbazone. the compounds (6a-g) form a new compounds have a new ring led to clear the antimicrobial activity, showed a variable degree of antibacterial activity against $E$. Coli and $S$. aureus.

\subsection{Antimicrobial activity}

The antibacterial and antifungal activities of the newly synthesized compounds were also carried out (in the Microbiology Division, Microanalytical Center, Cairo University) using the Diffusion Plate Method, DPM (Solomons and Doorenbos,1974,Vargha et al.,1949). A bottomless cylinder containing a measured quantity of 
the sample $(1 \mathrm{~mL}, 20 \mathrm{mg} / \mathrm{mL})$ is placed on a plate $(7 \mathrm{~cm}$ diameter) containing a solid bacterial medium (nutrient agar) or a fungal medium (Dox's medium) which has been heavily seeded with the spore suspension of the test organism. After incubation ( $24 \mathrm{~h}$ for bacteria and 5 days for fungi), the diameter of the clear zone of inhibition surrounding the sample is taken as a measure of the inhibitory power of the sample against the particular test organism $(\%$ inhibition $=$ sample inhibition zone $(\mathrm{cm}) /$ std. inhibition zone $(\mathrm{cm}) \times 100)$. All measurements were done in DMSO as a solvent which has zero inhibition activity. The obtained results were compared with reference antibiotic that were purchased from the Egyptian market (Amoxicillin trihydrate). As shown from the Table I , all tested compounds were found to low exhibit activity against each of Escherichia coli and Staphylococcus aureus microorganisms with respect to the used reference amoxicillin. The highlight is that the four compounds $6 \mathrm{c}, 6 \mathrm{~d}, 6 \mathrm{e}$ and 7 were more effective with comparison to standard from other compounds. The antifungal activity of all the tested compounds was found to be negative results.

Structural activity relationship (SAR)

The structural activity relationship (SAR) of newly synthesized compounds 5a-g, 6a-g and 7 explored the importance of the planer bicyclic benzofuran system in antibacterial inhibition activity in vitro. In general the presence of lipophilic function (LF) at position 6 such as methyl, ethyl, propyl, allyl and benzyl groups had low effect on the antibacterial activity. Also, there is difference in the biological profile between thiosemicarbazone derivatives 5 and triazole derivatives 6 , which proof that benzofuran moiety is the main part in the biological activities and/ or the active site of receptor is too big. Finally, all the tested compounds showed no activity against A. flavus and C. albicans fungi.

\section{Experimental}

Melting points were measured with a Gallenkamp apparatus and are uncorrected. IR spectra were recorded on Bruker Vector 22 FTIR spectrophotometer. ${ }^{1} \mathrm{H}-\mathrm{NMR}$ spectra were determined in DMSO- $\mathrm{d}_{6}$ and $\mathrm{CDCl}_{3}$ at 300 $\mathrm{MHz}$ on Varian Mercury VX spectrometer using TMS as an internal standard. Chemical shifts are expressed as $\delta$ ppm units. Mass spectra were recorded on GC-MS QP 1000 EX spectrometer at $70 \mathrm{eV}$. Elemental analyses were carried out at the Microanalytical Center of Cairo University. In all the ${ }^{1} \mathrm{H}-\mathrm{NMR}$ spectra $\left(^{*}\right)$ means that the signal is lost after $\mathrm{D}_{2} \mathrm{O}$ exchange.

\subsection{Synthesis of the alkyl and aralkyl ethers (3a-g):}

\section{General Procedure}

A solution of visnaginone $(2,10 \mathrm{~g})$ in methanolic sodium methoxide ( $1.15 \mathrm{~g}$ sodium metal in $50 \mathrm{ml}$ methanol) was heated under reflux on the steam-bath for 5 minutes. To this solution, the equimolecular amount of the alkyl or aralkyl chloride was added gradually and heating was continued until the reaction mixture became either neutral or acidic to litmus. The solvent was distilled off, and the residue filtered off, washed with $5 \%$ aqueous sodium hydroxide and then water until alkali-free. The reaction product was then crystallized from methanol to give (3a-g).

The IR spectrum of the reaction product showed the absorption band of acetyl-CO group at $\left(1720 \mathrm{~cm}^{-1}\right)$ in its IR spectrum. Its ${ }^{1} \mathrm{H}-\mathrm{NMR}$ spectrum revealed the presence of $2.55\left(3 \mathrm{H}, s\right.$, acetyl- $\mathrm{CH}_{3}$ group), $3.73\left(6 \mathrm{H}, s\right.$, two $\mathrm{CH}_{3} \mathrm{O}$ groups), $6.6(1 \mathrm{H}, s$, aromatic- $\mathrm{CH}), 6.66(1 \mathrm{H}, d$, furan $\mathrm{H}-3)$ in addition to furan $\mathrm{H}-2$ as a doublet at $7.52 \delta \mathrm{ppm})$. The reaction product could then be formulated as 1(4,6-dimethoxyben-zofuran-5-yl) ethanone (visnaginonemethylether, $3 \mathrm{a}$ ).

In a similar manner, there could be obtained 1(4-methoxy-6-ethoxyenzofuran-5-yl) ethanone (visnaginone-ethyl ether, 3b), 1(4-methoxy-4-n-propoxybenzo- furan -5-yl) ethanone(visnaginone-n-propyl ether 3c),1(4-methoxy-6-isopropoxybenzofuran-5-yl) ethanone(visnaginone-isopropylether, 3d), 1(4-methoxy-6-n-butoxyxybenzofuran-5-yl)ethanone(visnaginone-n-butylether,3e),

1(4-methoxy-6-allyloxybenzofuran-5-yl)ethanone (visnaginone-allyl ether, 3f) and 1(4-methoxy-6-benzyloxy-benzofuran-5-yl) ethanone (visnaginone-benzyl ether, 3g).

The structure of each of ( $3 \mathrm{~b}-\mathrm{g})$ was also established based on elemental analyses and spectral data studies. The mass spectrum of (3f) gave $\mathrm{m} / \mathrm{e}=246(100 \%)$ which is the exact mass required for the formula $\mathrm{C}_{14} \mathrm{H}_{14} \mathrm{O}_{4}$ assigned for the compound (cf. Experimental Part).

It has been found that the alkyl and aralkyl ethereal groups at position 6 in each of (3a-g) underwent preferential cleavage on boiling their solutions in acetic-hydrochloric acid mixture for 5 minutes. Visnaginone was the only isolated reaction product in all cases, although the isopropyl ether derivative was the first to be cleaved after two minutes only (TLC monitoring, cf. Experimental Part). 
A positive ferric chloride test was found to be + ve after the cleavage process in each case.

1-(4,6-dimethoxy-1-benzofuran-5-yl)ethanone (visnaginone-methyl ether), (3a):

was separated as colourless crystals with m.p. and mixed m.p. $135^{\circ} \mathrm{C}$.

1-(6-ethoxy-4-methoxy-1-benzofuran-5-yl)ethanone - ethane (1:1) (visnaginone-ethyl ether, (3b):

was separated as colourless crystals with m.p. and mixed m.p.152-153 ${ }^{\circ} \mathrm{C}$ (Spath and Gruber,1941).

1-(4-methoxy-6-propoxy-1-benzofuran-5-yl)ethanone (visnaginone-n-propylether, (3c):

was separated as yellow oil with b.p. $218^{\circ} \mathrm{C}(4 \mathrm{~mm})$ which solidified on standing to pale yellow crystals with m.p. and mixed m.p. $38^{\circ} \mathrm{C}$. (Anteunis et al.,1972).

1-(4-methoxy-6-(propan-2-yloxy)-1-benzofuran-5-yl)ethanone (visnaginone-isopropylether, (3d)

was separated as yellow oil with b.p. $205^{\circ} \mathrm{C}(1.5 \mathrm{~mm})$ which solidified on standing to pale yellow crystals with m.p. and mixed m.p. $35^{\circ} \mathrm{C}$. (Anteunis et al.,1972).

1-(6-butoxy-4-methoxy-1-benzofuran-5-yl)ethanone (visnaginone-n-butylether, (3e)

was separated as yellow oil with b.p. $225^{\circ} \mathrm{C}(5.5 \mathrm{~mm})$ which solidified on standing to pale yellow crystals with m.p. and mixed m.p. $42^{\circ} \mathrm{C}$. (Anteunis et al.,1972).

1-(4-methoxy-6-(prop-2-en-1-yloxy)-1-benzofuran-5-yl)ethanone (visnaginone-allyl ether) (3f):

was separated as yellow oil with b.p. $215^{\circ} \mathrm{C}(3.5 \mathrm{~mm})$ which solidified on standing to pale yellow crystals $(82 \%$ yield) with m.p. $48^{\circ} \mathrm{C}$, Anal. Calcd. for $\mathrm{C}_{14} \mathrm{H}_{14} \mathrm{O}_{4}: \quad$ C, 68.28, H, 5.73. Found: $\mathrm{C}, 68.35, \mathrm{H}, 5.80$, IR $\left(\mathrm{KBr}, \mathrm{cm}^{-1}\right)$ : sat-CH $\left(2980 \mathrm{~cm}^{-1}\right)$, acetyl-CO $\left(1720 \mathrm{~cm}^{-1}\right)$ and $\mathrm{C}=\mathrm{C}\left(1630 \mathrm{~cm}^{-1}\right),{ }^{1} \mathrm{H}-\mathrm{NMR}$ (DMSO- $\left.d_{6}, \delta / \mathrm{ppm}\right): 2.55(3 \mathrm{H}, s$, acetyl- $\mathrm{CH}_{3}$ group), $3.73\left(6 \mathrm{H}, s\right.$, two $\mathrm{CH}_{3} \mathrm{O}$ groups), $4.61\left(2 \mathrm{H}, d\right.$, allyl- $\left.\mathrm{CH}_{2}\right), 5.23(1 \mathrm{H}, s$, allyl-CH), $5.24(1 \mathrm{H}, d$, allyl-CH), $5.89(1 \mathrm{H}, m$, allyl-CH), $6.64(1 \mathrm{H}, s$, aromatic- $\mathrm{CH}), 6.66(1 \mathrm{H}, d$, furan $\mathrm{H}-3), 7.52(1 \mathrm{H}, d$, furan $\mathrm{H}-2)$, Mass spectra: $m / e=246(100 \%)$.

1-[6-(benzyloxy)-4-methoxy-1-benzofuran-5-yl]ethanone (visnaginone benzylether) (3g):

was separated as yellowish-brown oil with b.p. $230^{\circ} \mathrm{C}$. (Anteunis et al.,1972). (6.5 mm) which did not solidify on long standing.

3.2 Preferential dealkylation of ( $3 a-g)$ :

General Procedure :

A solution of the alkyl or aralkyl ether (3a-g), $2 \mathrm{~g}$ in a mixture of glacial acetic acid (15 ml) and 48\% $\mathrm{HCl}(3 \mathrm{ml})$ was heated on the water-bath for $5 \mathrm{~min}$. and then diluted with water. The solid obtained was filtered off, washed with water till acid-free then crystallized from methanol to give visnaginone in each case with m.p. and mixed m.p. 207-208 ${ }^{\circ}$ C. (Anteunis et al.,1972).

\subsection{Synthesis of the Thiosemicarbazones (5a-g):}

\subsubsection{Method A:}

A solution of the appropriate visnaginone alkyl or aralkyl ether (3a-g), $2 \mathrm{~g}$, methanolic sodium methoxide (prepared from the equivalent amount of sodium metal and methanol, $30 \mathrm{~mL}$ ) and sodium acetate $(1.8 \mathrm{~g}$ ) was heated on the water-bath for 15 minutes. The solid obtained on cooling was filtered off, washed with water then crystallized from methanol to give the corresponding (5a-f), (Scheme II).

(2E)-2-[1-(4,6-Dimethoxy-1-benzofuran-5-yl)ethylidene]hydrazinecarbothioamide (5a):

It separated from methanol as pale yellow crystals $\left(85 \%\right.$ yield) with m.p. $130^{\circ} \mathrm{C}$, Anal. Calcd. for $\mathrm{C}_{13} \mathrm{H}_{15} \mathrm{~N}_{3} \mathrm{O}_{3}: \mathrm{C}$, 53.23, H, 5.15, N, 14.32, S, 10.93. Found: C, 53.40, H, 5.20, N, 14.42, S, 10.74, IR (KBr, $\left.\mathrm{cm}^{-1}\right): 3389,3365,3350$, $3340\left(\mathrm{NH}\right.$ and $\left.\mathrm{NH}_{2}\right), 2980$ (sat-CH) and $1250(\mathrm{C}=\mathrm{S}),{ }^{1} \mathrm{H}-\mathrm{NMR}$ (DMSO- $d_{6}, \delta / p p m$ ): $0.9\left(3 \mathrm{H}, s\right.$, side chain- $\mathrm{CH}_{3}$ ), $2 *\left(2 \mathrm{H}, s, b r, \mathrm{NH}_{2}\right), 3.7\left(3 \mathrm{H}, s, \mathrm{OCH}_{3}\right.$ group), $3.75\left(3 \mathrm{H}, s, \mathrm{OCH}_{3}\right.$ group $), 6.5(1 \mathrm{H}, s$, aromatic-CH), $6.7(1 \mathrm{H}, d$, furan $\mathrm{H}-3), 7.4(1 \mathrm{H}, d$, furan $\mathrm{H}-2), 7.0 *(2 \mathrm{H}, \mathrm{s}, \mathrm{NH})$, Mass spectra, $m / e=293(78 \%)$.

(2E)-2-[1-(6-Ethoxy-4-methoxy-1-benzofuran-5-yl)ethylidene]hydrazine carbothioamide (5b):

It separated from methanol as pale yellow crystals ( $75 \%$ yield) with m.p. $167^{\circ} \mathrm{C}$, Anal. Calcd. for $\mathrm{C}_{14} \mathrm{H}_{17} \mathrm{~N}_{3} \mathrm{O}_{3} \mathrm{~S}: \mathrm{C}$, 54.71, H, 5.57, N, 13.67, S, 10.43. Found: $\quad$ C, 54.50, H, 5.35, N, 13.52 S, 10.34. IR $\left(\mathrm{KBr}, \mathrm{cm}^{-1}\right): 3380,3355$, 3350, $3340\left(\mathrm{NH}\right.$ and $\left.\mathrm{NH}_{2}\right), 2990(\mathrm{sat}-\mathrm{CH})$ and $1260(\mathrm{C}=\mathrm{S}) .{ }^{1} \mathrm{H}-\mathrm{NMR}$ (DMSO- $\left.d_{6}, \delta / p p m\right): 1.0(3 \mathrm{H}, s$, side chain- $\left.\mathrm{CH}_{3}\right), 1.3\left(5 \mathrm{H}, t, \mathrm{CH}_{3} \mathrm{CH}_{2}\right), 2.2 *\left(2 \mathrm{H}, s, b r, \mathrm{NH}_{2}\right), 3.8\left(3 \mathrm{H}, s, \mathrm{OCH}_{3}\right), 4.1\left(5 \mathrm{H}, q, \mathrm{CH}_{3} \mathrm{CH}_{2}\right), 6.4(1 \mathrm{H}, s$, aromatic-CH), $6.8(1 \mathrm{H}, d$, furan $\mathrm{H}-3), 7.5(1 \mathrm{H}, d$, furan $\mathrm{H}-2), 7.0 *(1 \mathrm{H}, s, b r, \mathrm{NH})$, Mass spectra, $m / e=307(62 \%)$. 
(2E)-2-[1-(4-Methoxy-6-propoxy-1-benzofuran-5-yl)ethylidene]hydrazine carbothioamide (5c):

It was separated from methanol as pale yellow crystals $(81 \%$ yield $)$ with m.p. $164^{\circ} \mathrm{C}$. Anal. Calcd. for $\mathrm{C}_{15} \mathrm{H}_{19} \mathrm{~N}_{3} \mathrm{O}_{3} \mathrm{~S}$ : C, 56.06, H, 5.96, N, 13.07, S, 9.98. Found: $\quad \mathrm{C}, 56.20, \mathrm{H}, 5.75, \mathrm{~N}, 13.22, \mathrm{~S}, 9.84, \mathrm{IR}(\mathrm{KBr}$, $\left.\mathrm{cm}^{-1}\right): 3370,3350,3345,3330\left(\mathrm{NH}\right.$ and $\left.\mathrm{NH}_{2}\right), 2995(\mathrm{sat}-\mathrm{CH})$ and $1255(\mathrm{C}=\mathrm{S}) .{ }^{1} \mathrm{H}-\mathrm{NMR}$ (DMSO- $\left.d_{6}, \delta / \mathrm{ppm}\right): 0.95$ $\left(3 \mathrm{H}, s\right.$, side chain- $\left.\mathrm{CH}_{3}\right), 1\left(7 \mathrm{H}, t, \mathrm{CH}_{3} \mathrm{CH}_{2} \mathrm{CH}_{2}\right), 1.75\left(7 \mathrm{H}, m, \mathrm{CH}_{3} \mathrm{CH}_{2} \mathrm{CH}_{2}\right), 2.2^{*}\left(2 \mathrm{H}, s, b r, \mathrm{NH}_{2}\right), 3.85(3 \mathrm{H}, s$, $\left.\mathrm{OCH}_{3}\right), 3.94\left(7 \mathrm{H}, q, \mathrm{CH}_{3} \mathrm{CH}_{2} \mathrm{CH}_{2}\right), 6.4\left(1 \mathrm{H}, s\right.$, aromatic-CH), 6,8 $(1 \mathrm{H}, d$, furan $\mathrm{H}-3), 7.4(1 \mathrm{H}, d$, furan $\mathrm{H}-2), 7.8^{*}$ $(1 \mathrm{H}, s, b r, \mathrm{NH})$, Mass spectra, $m / e=321(77 \%)$.

(2E)-2-\{1-[4-Methoxy-6-(propan-2-yloxy)-1-benzofuran-5-yl]ethylidene $\}$ hydrazine carbothioamide (5d):

It separated from methanol as light brown crystals ( $83 \%$ yield) with m.p. $159^{\circ} \mathrm{C}$. Anal. Calcd. for $\mathrm{C}_{15} \mathrm{H}_{19} \mathrm{~N}_{3} \mathrm{O}_{3} \mathrm{~S}: \mathrm{C}$, 56.06, H, 5.96, N, 13.07, S, 9.98. Found: C, 56.30, H, 5.70, N, 13.20, S, 9.78, IR $\left(\mathrm{KBr}, \mathrm{cm}^{-1}\right): 3360,3355$, 3340, $3320\left(\mathrm{NH}\right.$ and $\left.\mathrm{NH}_{2}\right), 2980\left(\right.$ sat-CH) and $1250(\mathrm{C}=\mathrm{S}) .{ }^{1} \mathrm{H}-\mathrm{NMR}\left(\mathrm{DMSO}-\mathrm{d}_{6}, \delta / \mathrm{ppm}\right): 0.9(3 \mathrm{H}$, s, side chain- $\left.\mathrm{CH}_{3}\right), 1.38\left(7 \mathrm{H}, \mathrm{dd}, \mathrm{CH}\left(\mathrm{CH}_{3}\right)_{2}\right), 2.1 *\left(2 \mathrm{H}, \mathrm{s}, \mathrm{br}, \mathrm{NH}_{2}\right), 3.8\left(3 \mathrm{H}, \mathrm{s}, \mathrm{OCH}_{3}\right), 4.04\left(7 \mathrm{H}, \mathrm{m}, \mathrm{CH}\left(\mathrm{CH}_{3}\right)_{2}\right), 6.5(1 \mathrm{H}$, $\mathrm{s}$, aromatic-CH), $6.8(1 \mathrm{H}, \mathrm{d}$, furan $\mathrm{H}-3), 7.6(1 \mathrm{H}, \mathrm{d}$, furan $\mathrm{H}-2), 8.0^{*}(1 \mathrm{H}, \mathrm{s}, \mathrm{br}, \mathrm{NH})$, Mass spectra, $\mathrm{m} / \mathrm{e}=321$ $(84 \%)$.

(2E)-2-[1-(6-Butoxy-4-methoxy-1-benzofuran-5-yl)ethylidene]hydrazinecarbothioamide (5e):

It separated from methanol as light brown crystals $\left(78 \%\right.$ yield) with m.p. $123^{\circ} \mathrm{C}$.

Anal. Calcd. for $\mathrm{C}_{16} \mathrm{H}_{21} \mathrm{~N}_{3} \mathrm{O}_{3} \mathrm{~S}$ : C, 57.29, H, 6.31, N, 12.53, S, 9.56. Found: C, 57.35, H, 6.40, N, 13.35, S, 9.70, IR $\left(\mathrm{KBr}, \mathrm{cm}^{-1}\right): 3365,3355,3340,3320\left(\mathrm{NH}\right.$ and $\left.\mathrm{NH}_{2}\right), 2985(\mathrm{sat}-\mathrm{CH})$ and $1260(\mathrm{C}=\mathrm{S}) .{ }^{1} \mathrm{H}-\mathrm{NMR}$ (DMSO- $d_{6}, \delta /$ ppm ): 0.95 (3H, s, side chain- $\left.\mathrm{CH}_{3}\right), 1.0\left(9 \mathrm{H}, t, \mathrm{CH}_{2} \mathrm{CH}_{2} \mathrm{CH}_{2} \mathrm{CH}_{3}\right), 1.33\left(9 \mathrm{H}, m, \mathrm{CH}_{2} \mathrm{CH}_{2} \mathrm{CH}_{2} \mathrm{CH}_{3}\right), 1.7(9 \mathrm{H}, m$, $\left.\mathrm{CH}_{2} \mathrm{CH}_{2} \mathrm{CH}_{2} \mathrm{CH}_{3}\right), 2.1^{*}\left(2 \mathrm{H}, s, b r, \mathrm{NH}_{2}\right), 3.8\left(3 \mathrm{H}, s, \mathrm{OCH}_{3}\right), 3.9\left(9 \mathrm{H}, d d, \mathrm{CH}_{2} \mathrm{CH}_{2} \mathrm{CH}_{2} \mathrm{CH}_{3}\right), 6.6(1 \mathrm{H}, s$, aromatic-CH), $6.9(1 \mathrm{H}, d$, furan $\mathrm{H}-3), 7.4(1 \mathrm{H}, d$, furan $\mathrm{H}-2), 7.95^{*}(1 \mathrm{H}, s, b r, \mathrm{NH})$, Mass spectra, $m / e=335$ $(62 \%)$.

(2E)-2-\{1-[4-Methoxy-6-(prop-2-en-1-yloxy)-1-benzofuran-5-yl]ethylidene $\}$ hydrazinecarbothioamide (5f):

It separated from methanol as light yellow crystals $\left(73 \%\right.$ yield) with m.p. $105^{\circ} \mathrm{C}$. Anal. Calcd. for $\mathrm{C}_{15} \mathrm{H}_{17} \mathrm{~N}_{3} \mathrm{O}_{3} \mathrm{~S}$ : C, 56.41, H, 5.37, N, 13.16, S, 10.04. Found: C, 56.60, H, 5.40, N, 13.35, S, 10.20, IR (KBr, cm $\left.{ }^{-1}\right): 3365,3350$, 3340, $3330\left(\mathrm{NH}\right.$ and $\mathrm{NH}_{2}$ ), $2980\left(\right.$ sat-CH) and $1250(\mathrm{C}=\mathrm{S}) .{ }^{1} \mathrm{H}-\mathrm{NMR}$ (DMSO- $\left.d_{6}, \delta / p p m\right): 0.90(3 \mathrm{H}, s$, side chain- $\left.\mathrm{CH}_{3}\right), 2.3 *\left(2 \mathrm{H}, s, b r, \mathrm{NH}_{2}\right), 3.95\left(3 \mathrm{H}, s, \mathrm{OCH}_{3}\right), 4.6\left(5 \mathrm{H}, d d, \mathrm{CH}_{2} \mathrm{CH}=\mathrm{CH}_{2}\right), 5.23\left(5 \mathrm{H}, d d, \mathrm{CH}_{2} \mathrm{CH}=\mathrm{CH}_{2}\right)$, $5.24\left(5 \mathrm{H}, d d, \mathrm{CH}_{2} \mathrm{CH}=\mathrm{CH}_{2}\right), 5.9\left(5 \mathrm{H}, m, \mathrm{CH}_{2} \mathrm{CH}=\mathrm{CH}_{2}\right), 6.5(1 \mathrm{H}, s$, aromatic- $\mathrm{CH}), 6.8(1 \mathrm{H}, d$, furan $\mathrm{H}-3), 7.6(1 \mathrm{H}$, $d$, furan $\mathrm{H}-2), 8.1^{*}(1 \mathrm{H}, s, b r, \mathrm{NH})$, Mass spectra, $m / e=319(77 \%)$.

(2E)-2-\{1-[6-(Benzyloxy)-4-methoxy-1-benzofuran-5-yl]ethylidene $\}$ hydrazine carbothioamide (5g):

It separated from methanol as yellowish-green crystals $(82 \%$ yield $)$ with m.p. $119^{\circ} \mathrm{C}$. Anal. Calcd. for $\mathrm{C}_{19} \mathrm{H}_{19} \mathrm{~N}_{3} \mathrm{O}_{3} \mathrm{~S}: \mathrm{C}, 61.77, \mathrm{H}, 5.18, \mathrm{~N}, 11.37, \mathrm{~S}, 8.68$. Found : C, 61.60, H, 5.30, N, 11.35, S, 8.90, IR $\left(\mathrm{KBr}, \mathrm{cm}^{-1}\right)$ : $3365,3350,3345,3330\left(\mathrm{NH}\right.$ and $\left.\mathrm{NH}_{2}\right), 2990$ (sat-CH) and $1250(\mathrm{C}=\mathrm{S}) .{ }^{1} \mathrm{H}-\mathrm{NMR}$ (DMSO- $\left.d_{6}, \delta / \mathrm{ppm}\right): 1.0(3 \mathrm{H}, s$, side chain- $\left.\mathrm{CH}_{3}\right), 2.1^{*}\left(2 \mathrm{H}, s, b r, \mathrm{NH}_{2}\right), 3.85\left(3 \mathrm{H}, s, \mathrm{OCH}_{3}\right), 5.2\left(7 \mathrm{H}, d, \mathrm{CH}_{2}-\mathrm{C}_{6} \mathrm{H}_{5}\right), 6.5(1 \mathrm{H}, s$, aromatic-CH), 6.8 $(1 \mathrm{H}, d$, furan $\mathrm{H}-3), 7.1-7.4\left(7 \mathrm{H}, m, \mathrm{CH}_{2}-\mathrm{C}_{6} \mathrm{H}_{5}\right), 7.6(1 \mathrm{H}, d$, furan $\mathrm{H}-2), 8.1^{*}(1 \mathrm{H}, s, b r, \mathrm{NH})$, Mass spectra, $m / e=$ $359(67 \%)$.

\subsubsection{Method B:}

A solution of $7(0.01 \mathrm{~mole})$ in methanol $(30 \mathrm{~mL})$ containing sodium acetate $(1.5 \mathrm{~g})$ and the appropriate aryl or aralkyl iodide $(0.01 \mathrm{~mol})$ was heated on the water-bath for 30 minutes. The reaction mixture was heated with $20 \%$ sodium hydroxide solution for 30 minutes then poured onto cold water. The solid obtained was filtered off, washed with water then crystallized from methanol to give(5a-g) respectively with the same physical, analytical and spectral data as (5a-g) prepared as described in method A, ( Scheme II ).

\subsection{Synthesis of the 1,2,4-triazole derivatives (6a-g):}

\subsubsection{Method A:}

A mixture of each of $(3 \mathrm{a}-\mathrm{g})(0.01 \mathrm{~mol})$ and thiosemicarbazide hydrochloride $(0.01 \mathrm{~mole})$ in methanol $(30 \mathrm{~mL})$ and hydrochloric acid $(1.5 \mathrm{~mL})$ was heated on the water-bath for 30 minutes. The solid obtained after cooling was filtered off, washed with water till acid-free then crystallized from methanol to give (6a-g) respectively:

\section{5-(4,6-Dimethoxy-1-benzofuran-5-yl)-5-methyl-1,2,4-triazoline-3-thione (6a):}

It separated as brown crystals with m.p. $161{ }^{\circ} \mathrm{C}\left(64 \%\right.$ yield). Anal. Calcd. for $\mathrm{C}_{13} \mathrm{H}_{15} \mathrm{~N}_{3} \mathrm{O}_{3} \mathrm{~S}: \mathrm{C}, 53.23, \mathrm{H}, 5.15, \mathrm{~N}$, 14.32, S, 10.93. Found : C, 53.28, H, 5.22, N, 14.44, S, 10.83, IR $\left(\mathrm{KBr}, \mathrm{cm}^{-1}\right)$ : three NH $(3360,3345,3340)$, sat-CH (2970) and ring-C $=\mathrm{S}(2720) .{ }^{1} \mathrm{H}-\mathrm{NMR}\left(\mathrm{DMSO}-d_{6,} \delta / \mathrm{ppm}\right): 2.0^{*}(1 \mathrm{H}, s s s, b r, \mathrm{NH}), 2.1 *(1 \mathrm{H}, s s s, b r, \mathrm{NH})$, 
2.4* (1H, sss, br, NH), 3.73 (3H, s,s, $\mathrm{CH}_{3} \mathrm{O}$ groups), 3.73 (3H, s,s, $\mathrm{CH}_{3} \mathrm{O}$ groups), 6.67 (1H, s, aromatic- $\left.\mathrm{CH}\right), 6.66$ $(1 \mathrm{H}, d$, furan $\mathrm{H}-3), 7.52(1 \mathrm{H}, d$, furan $\mathrm{H}-2)$, Mass spectra: $m / e=293(56 \%)$.

5-(6-Eethoxy-4-methoxy-1-benzofuran-5-yl)-5-methyl-1,2,4-triazolidine-3-thione (6b):

It separated as light brown crystals with m.p. $190{ }^{\circ} \mathrm{C}(75 \%$ yield $)$.

Anal. Calcd. for $\mathrm{C}_{14} \mathrm{H}_{17} \mathrm{~N}_{3} \mathrm{O}_{3} \mathrm{~S}: \mathrm{C}, 54.00, \mathrm{H}, 5.42, \mathrm{~N}, 13.44, \mathrm{~S}, 10.60$. Found: C, 54.71, H, 5.57, N, 13.67, S, 10.43 , IR $\left(\mathrm{KBr}, \mathrm{cm}^{-1}\right)$ : three $\mathrm{NH}(3350,3340,3330)$, sat-CH $(2980)$ and ring-C=S (2700). ${ }^{1} \mathrm{H}-\mathrm{NMR}$ (DMSO- $d_{6}, \delta /$ ppm ): $1.33\left(5 \mathrm{H}, t, \mathrm{CH}_{2} \mathrm{CH}_{3}\right), 2.0 *(1 \mathrm{H}, s s s, b r, \mathrm{NH}), 2.1 *(1 \mathrm{H}, s s s, b r, \mathrm{NH}), 2.3 *(1 \mathrm{H}, s s s, b r, \mathrm{NH}), 1.52(3 \mathrm{H}, s$, $\mathrm{CH}_{3}$ group), $3.73\left(3 \mathrm{H}, s, s, \mathrm{CH}_{3} \mathrm{O}\right.$ groups $), 3.98\left(5 \mathrm{H}, q, \mathrm{CH}_{2} \mathrm{CH}_{3}\right), 6.64(1 \mathrm{H}, s$, aromatic- $\mathrm{CH}), 6.66(1 \mathrm{H}, d$, furan $\mathrm{H}-3), 7.52(1 \mathrm{H}, d$, furan $\mathrm{H}-2)$, Mass spectra, $m / e=307$.

5-(4-Methoxy-6-propoxy-1-benzofuran-5-yl)-5-methyl-1,2,4-triazolidine-3-thione (6c):

It separated as light brown crystals with m.p. $157^{\circ} \mathrm{C}(88 \%$ yield $)$.

Anal. Calcd. for $\mathrm{C}_{15} \mathrm{H}_{19} \mathrm{~N}_{3} \mathrm{O}_{3} \mathrm{~S}$ : C, 56.06, H, 5.96, N, 13.07, S, 9.98. Found: C, 56.15, H, 5.80, N, 13.20, S, 9.80, IR $\left(\mathrm{KBr}, \mathrm{cm}^{-1}\right)$ : Three $\mathrm{NH}(3355,3340,3335)$, sat-CH (2995) and ring-C $=\mathrm{S}(2750) .{ }^{1} \mathrm{H}-\mathrm{NMR}\left(\mathrm{CDCl}_{3}, \delta / \mathrm{ppm}\right): 0.90$ $\left(3 \mathrm{H}, s\right.$, side chain- $\left.\mathrm{CH}_{3}\right), 1.1\left(7 \mathrm{H}, t, \mathrm{CH}_{3} \mathrm{CH}_{2} \mathrm{CH}_{2}\right), 1.70\left(7 \mathrm{H}, m, \mathrm{CH}_{3} \mathrm{CH}_{2} \mathrm{CH}_{2}\right), 2.0 *(1 \mathrm{H}, s s s, b r, \mathrm{NH}), 2.2 *(1 \mathrm{H}, s s s$, $b r, \mathrm{NH}), 2.3 *(1 \mathrm{H}, s s s, b r, \mathrm{NH}), 3.95\left(3 \mathrm{H}, s, \mathrm{OCH}_{3}\right), 4.1\left(7 \mathrm{H}, q, \mathrm{CH}_{3} \mathrm{CH}_{2} \mathrm{CH}_{2}\right), 6.5(1 \mathrm{H}, s$, aromatic- $\mathrm{CH}), 6.7(1 \mathrm{H}$, $d$, furan $\mathrm{H}-3), 7.3(1 \mathrm{H}, d$, furan $\mathrm{H}-2)$, Mass spectra, $m / e=321(68 \%)$.

5-[4-Methoxy-6-(propan-2-yloxy)-1-benzofuran-5-yl]-5-methyl-1,2,4-triazolidine-3-thione (6d):

It separated as light brown crystals with m.p. $167{ }^{\circ} \mathrm{C}(76 \%$ yield $)$.

Anal. Calcd. for $\mathrm{C}_{15} \mathrm{H}_{19} \mathrm{~N}_{3} \mathrm{O}_{3} \mathrm{~S}$ : C, 56.06, H, 5.96, N, 13.07, S, 9.98. Found: C, 56.15, H, 5.80, N, 13.00, S, 9.80, IR $\left(\mathrm{KBr}, \mathrm{cm}^{-1}\right)$ : Three NH $(3455,3400,3335)$, sat-CH (2900) and ring-C $=\mathrm{S}(2750) .{ }^{1} \mathrm{H}-\mathrm{NMR}\left(\mathrm{DMSO}-d_{6}, \delta / \mathrm{ppm}\right)$ : $1.1\left(3 \mathrm{H}, s\right.$, side chain- $\left.\mathrm{CH}_{3}\right), 1.35\left(7 \mathrm{H}, t w o d d, \mathrm{CH}\left(\mathrm{CH}_{3}\right)_{2}\right), 2.2 *(1 \mathrm{H}, s s s, b r, \mathrm{NH}), 2.3 *(1 \mathrm{H}, s s s, b r, \mathrm{NH}), 2.45^{*}(1 \mathrm{H}$, $s s s, b r, \mathrm{NH}), \quad 3.9\left(3 \mathrm{H}, s, \mathrm{OCH}_{3}\right), 4.15\left(7 \mathrm{H}, m, \mathrm{CH}\left(\mathrm{CH}_{3}\right)_{2}\right), 6.4(1 \mathrm{H}, s$, aromatic- $\mathrm{CH}), 6.7(1 \mathrm{H}, d$, furan $\mathrm{H}-3), 7.5$ $(1 \mathrm{H}, d$, furan $\mathrm{H}-2)$, Mass spectra, $m / e=321(66 \%)$.

5-(6-Butoxy-4-methoxy-1-benzofuran-5-yl)-5-methyl-1,2,4-triazolidine-3-thione (6e):

It separated as pale yellow crystals with m.p. $146^{\circ} \mathrm{C}$ ( $72 \%$ yield).

Anal. Calcd. for $\mathrm{C}_{16} \mathrm{H}_{21} \mathrm{~N}_{3} \mathrm{O}_{3} \mathrm{~S}: \mathrm{C}, 57.29, \mathrm{H}, 6.31, \mathrm{~N}, 12.53$, S, 9.56. Found: C, 57.40, H, 6.35, N, 13.40, S, 9.40, IR $\left(\mathrm{KBr}, \mathrm{cm}^{-1}\right)$ : Three NH $(3450,3400,3330)$, sat-CH (2950) and ring $-\mathrm{C}=\mathrm{S}(2710) .{ }^{1} \mathrm{H}-\mathrm{NMR}$ (DMSO- $\left.d_{6}, \delta / \mathrm{ppm}\right)$ : $1.0\left(3 \mathrm{H}, s\right.$, side chain- $\left.\mathrm{CH}_{3}\right), 1.1\left(9 \mathrm{H}, t, \mathrm{CH}_{2} \mathrm{CH}_{2} \mathrm{CH}_{2} \mathrm{CH}_{3}\right), 1.33\left(9 \mathrm{H}, m, \mathrm{CH}_{2} \mathrm{CH}_{2} \mathrm{CH}_{2} \mathrm{CH}_{3}\right), 1.75(9 \mathrm{H}, m$, $\left.\mathrm{CH}_{2} \mathrm{CH}_{2} \mathrm{CH}_{2} \mathrm{CH}_{3}\right), 2.2^{*}(1 \mathrm{H}, s s s, b r, \mathrm{NH}), 2.3^{*}(1 \mathrm{H}, s s s, b r, \mathrm{NH}), 2.5^{*}(1 \mathrm{H}, s s s, b r, \mathrm{NH}), 3.9\left(3 \mathrm{H}, s, \mathrm{OCH}_{3}\right), 4.15$ $\left(9 \mathrm{H}, d d, \mathrm{CH}_{2} \mathrm{CH}_{2} \mathrm{CH}_{2} \mathrm{CH}_{3}\right), 6.5(1 \mathrm{H}, s$, aromatic- $\mathrm{CH}), 6.7(1 \mathrm{H}, d$, furan $\mathrm{H}-3), 7.6(1 \mathrm{H}, d$, furan $\mathrm{H}-2)$, Mass spectra, $m / e=335(68 \%)$.

5-[4-Methoxy-6-(prop-2-en-1-yloxy)-1-benzofuran-5-yl]-5-methyl-1,2,4-triazolidine-3-thione (6f):

It separated as pale yellow crystals with m.p. $148{ }^{\circ} \mathrm{C}(62 \%$ yield $)$.

Anal. Calcd. for $\mathrm{C}_{15} \mathrm{H}_{17} \mathrm{~N}_{3} \mathrm{O}_{3} \mathrm{~S}: \mathrm{C}, 56.41, \mathrm{H}, 5.37, \mathrm{~N}, 13.16, \mathrm{~S}, 10.04$. Found: C, 56.35, H, 5.20, N, 13.25, S, 10.00, $\operatorname{IR}\left(\mathrm{KBr}, \mathrm{cm}^{-1}\right)$ : Three NH $(3400,3440,3330)$, sat-CH (2980) and ring-C=S (2710). ${ }^{1} \mathrm{H}-\mathrm{NMR}$ (DMSO- $\left.d_{6}, \delta / \mathrm{ppm}\right)$ : $0.90\left(3 \mathrm{H}, s\right.$, side chain- $\left.\mathrm{CH}_{3}\right), 2.2^{*}(1 \mathrm{H}, s s s, b r, \mathrm{NH}), 2.3^{*}(1 \mathrm{H}, s s s, b r, \mathrm{NH}), 2.4^{*}(1 \mathrm{H}, s s s, b r, \mathrm{NH}), 4.1(3 \mathrm{H}, s$, $\left.\mathrm{OCH}_{3}\right), 4.5\left(5 \mathrm{H}, d d, \mathrm{CH}_{2} \mathrm{CH}=\mathrm{CH}_{2}\right), 5.25\left(5 \mathrm{H}, d d, \mathrm{CH}_{2} \mathrm{CH}=\mathrm{CH}_{2}\right), 5.27\left(5 \mathrm{H}, d d, \mathrm{CH}_{2} \mathrm{CH} \mathrm{CH}_{2}\right), 5.85(5 \mathrm{H}, m$, $\left.\mathrm{CH}_{2} \mathrm{CH}=\mathrm{CH}_{2}\right), 6.6(1 \mathrm{H}, s$, aromatic- $\mathrm{CH}), 6.8(1 \mathrm{H}, d$, furan $\mathrm{H}-3), 7.5(1 \mathrm{H}, d$, furan $\mathrm{H}-2)$, Mass spectra, $m / e=319$ $(59 \%)$.

5-[6-(Benzyloxy)-4-methoxy-1-benzofuran-5-yl]-5-methyl-1,2,4-triazolidine-3-thione (6g):

It separated as pale yellow crystals with m.p. $162{ }^{\circ} \mathrm{C}(89 \%$ yield $)$.

Anal. Calcd. for $\mathrm{C}_{19} \mathrm{H}_{19} \mathrm{~N}_{3} \mathrm{O}_{3} \mathrm{~S}$ : C, 61.77, H, 5.18, N, 11.37, S, 8.68. Found : C, 61.70, H, 5.00, N, 11.25, S, 8.60, IR $\left(\mathrm{KBr}, \mathrm{cm}^{-1}\right)$ : Three NH $(3450,3410,3350)$, sat-CH (2985) and ring-C=S (2700). ${ }^{1} \mathrm{H}-\mathrm{NMR}$ (DMSO-d6, $\left.\delta / \mathrm{ppm}\right)$ : $1.0\left(3 \mathrm{H}, s\right.$, side chain- $\left.\mathrm{CH}_{3}\right), 2.1^{*}(1 \mathrm{H}, s s s, b r, \mathrm{NH}), 2.3 *(1 \mathrm{H}, s s s, b r, \mathrm{NH}), 2.4^{*}(1 \mathrm{H}, s s s, b r, \mathrm{NH}), 4.15(3 \mathrm{H}, s$, $\left.\mathrm{OCH}_{3}\right), 5.3\left(7 \mathrm{H}, d, \mathrm{CH}_{2}-\mathrm{C}_{6} \mathrm{H}_{5}\right), 6.6(1 \mathrm{H}, s$, aromatic- $\mathrm{CH}), 6.8(1 \mathrm{H}, d$, furan $\mathrm{H}-3), 7.1-7.4\left(7 \mathrm{H}, m, \mathrm{CH}_{2}-\mathrm{C}_{6} \mathrm{H}_{5}\right), 7.7$ $(1 \mathrm{H}, d$ furan $\mathrm{H}-2)$, Mass spectra, $m / e=359(69 \%)$.

\subsubsection{Method B:}

A solution of each of $(5 \mathrm{a}-\mathrm{g})(0.01 \mathrm{~mol})$ in methanol $(30 \mathrm{~mL})$ and hydrochloric acid $(1.5 \mathrm{~mL})$ was heated on the water-bath for 30 minutes. The solid obtained after cooling was filtered off, washed with water till acid-free then 
crystallized from methanol to give (6a-g) respectively. Compounds (6a-g) prepared via this route were found completely identical in analytical and spectral data as (6a-g) prepared as described before. (Scheme II).

\subsection{Synthesis of visnaginone thiosemicarbazone}

(2E)-2-[1-(6-Hydroxy-4-methoxy-1-benzofuran-5-yl)ethylidene]hydrazinecarbothioamide

(visnaginone thiosemicarbazone) (7):

A mixture of visnaginone ( $2 \mathrm{~g})$, thiosemicarbazide hydrochloride $(2 \mathrm{~g})$ and sodium acetate $(2 \mathrm{~g})$ in methanol (30 $\mathrm{mL}$ )was heated on the water0bath for 30 minutes. The solid obtained after cooling was filtered off, washed with water then crystallized from methanol to give

(2E)-2-[1-(6-hydroxy-4-methoxy-1-benzofuran-5-yl)ethylidene]hydrazinecarbothioamide(visnaginone thiosemicarbazone) (7), ( Scheme II ).

It separated as pale yellow crystals with m.p. $167^{\circ} \mathrm{C}\left(80 \%\right.$ yield). Anal. Calcd. for $\mathrm{C}_{12} \mathrm{H}_{13} \mathrm{~N}_{3} \mathrm{O}_{3} \mathrm{~S}: \mathrm{C}, 51.60, \mathrm{H}$, 4.69, N, 15.04, S, 11.48. Found: C, 51.80, H, 4.50, N, 15.20, S, 11.30, IR (KBr, cm $\left.{ }^{-1}\right): 3365,3350,3345,3330(\mathrm{NH}$ and $\left.\mathrm{NH}_{2}\right), 2980\left(\right.$ sat-CH) and $1260(\mathrm{C}=\mathrm{S}) .{ }^{1} \mathrm{H}-\mathrm{NMR}\left(\mathrm{DMSO}-d_{6}, \delta / \mathrm{ppm}\right): 1.0\left(3 \mathrm{H}, s\right.$, side chain- $\left.\mathrm{CH}_{3}\right), 2.1 *(2 \mathrm{H}, s$, $\left.b r, \mathrm{NH}_{2}\right), 3.85\left(3 \mathrm{H}, s, \mathrm{OCH}_{3}\right), 6.5(1 \mathrm{H}, s$, aromatic- $\mathrm{CH}), 6.8(1 \mathrm{H}, d$, furan $\mathrm{H}-3), 7.6(1 \mathrm{H}, d$, furan $\mathrm{H}-2), 8.1 *(1 \mathrm{H}, s$, $b r, \mathrm{NH}), 11.3(1 \mathrm{H}, s, \mathrm{OH})$, Mass spectra: $m / e=279(100 \%)$.

\section{References}

Achary, J.K and Rao N.A. (1992). A novel intermediate in the interaction of thiosemicarbazide with sheep liver serine hydroxyl methyltransferase. J. Biol. Chem. 267, 19066-19071. doi: http://www.jbc.org/content/267/27/19066.full.pdf

Anteunis, M., Borremans, F., Tadros, W, Zaher, A.H.A., and Chabrial, S.S. (1972). Isomerism of semicarbazones of 6-hydroxy-4,7-dimethoxy- and 6-hydroxy-4-methoxy-benzofuran-5-yl methyl ketone and derivatives J. Chem. Soc., Perkin Trans., 1, 616-619. doi:10.1039/P19720000616, http://dx.doi.org/10.1039/P19720000616

Bruneton, J. (1995). Pharmacognosy, Phytochemistry and Medicinal Plants, English Translation by Hatton, C. K., Lavoisier Publishing: Paris.

Chaudhary, S.K., Chaudhary, A., and Parmar S.S, (1978). Anticonvulsant and antiproteolytic properties of 3, 5-disubstituted oxadiazole-2-thiones and their inhibition of respiration in rat brain homogenates. J. Pharm. Sci., 67, 1507-1509. doi:10.1002/jps.2600671104, http://dx.doi.org/10.1002/jps.2600671104

Clarke, J. R., Robertson, A. (1949). Furano-compounds. Part IX. The synthesis of kellin and related compounds $J$. Chem. Soc., 302-307. doi:10.1039/JP9497300159, http://dx.doi.org/10.1039/JP9497300159

Geissmann, T. A., Halshall, T. G. (1951). Chromones. III. A Total Synthesis of Khellin1 J. Am. Chem. Soc., 73, 1280-1284. doi:10.1021/ja01147a121, http://dx.doi.org/10.1021/ja01147a121

Harborne, J. B., Williams, C. A. (2000). Advances in flavonoid research since 1992. Phytochemistry, 55, 481-504. doi:10.1016/S0031-9422(00)00235-1, http://dx.doi.org/10.1016/S0031-9422(00)00235-1

Heilbron, I. M., Barnes, H., Morton, R. A. (1923). Chemical reactivity and conjugation: the reactivity of the 2-methyl group in 2: 3-dimethylchromone J. Chem. Soc., Trans., 123, 2559 - 2570. doi:10.1039/CT9232302559, http://dx.doi.org/10.1039/CT9232302559

Hishmat, O.H., Atta S.M.S, and Abd-el Rahman A.H. (2002). Synthesis of Triazolyl-Oxadiazolyl-Thiazolyl- and Thiadiazolylbenzofuran of Potential Biological Activity Phosphorus, Sulfur, and Silicon and the Related Elements, 177, 863-875. doi:10.1080/10426500210654, http://dx.doi.org/10.1080/10426500210654

Huttrer C.P., and Dale, E.P. (1951). The Chemistry and Physiological Action of Khellin and Related Products, Chem. Rev.,48, 543-579. doi:10.1021/cr60151a003, http://dx.doi.org/10.1021/cr60151a003

Maria, B., Istvan H., Zoltan, M. and Levente, P. (1980). Studies on Chemotherapeutics III. Synthesis and Cyclisation of 5-Substituted-4-oxo-1,4-dihydro-3-pyridinecarbonylsemicarbazide and thiosemicarbazide $J$. Heterocyclic Chem., 17, 175-179. doi:10.1002/jhet.5570170134, http://dx.doi.org/10.1002/jhet.5570170134

Martinez-Utnilla, R., Miranda, M. A. (1981). Photochemistry of 5-aryl-2(3H)-furanones. : A new route to the synthesis of chromones Tetrahedron, 37, 2111-2114. doi:10.1016/S0040-4020(01)97966-9, http://dx.doi.org/10.1016/S0040-4020(01)97966-9

McClure, J. W., Harborne, J. B., Mabry, T. J., Mabry, H. (1975). The Flavonoids Eds, Chapman and Hall: London. Middleton, Jr. E., Kandaswami, C., Arborne, J. B. (1994). The Flavonoids Advances in Research Since 1986 Ed., Chapman and Hall : London. 
Rajanna, K. C. , Solomon, F. , Moazzam, M. A. and Saiprakash, P. K. (1996). Kinetics and mechanism of Vilsmeier-Haack synthesis of 3-formyl chromones derived from o-hydroxy aryl alkyl ketones: A structure reactivity study. Tetrahedron,52,3669-3682. doi:10.1016/0040-4020(96)00043-9, http://dx.doi.org/10.1016/0040-4020(96)00043-9

Rene, B. and Christophe, M. (1977). Action of hydroxylamine on chomone and Khellin. Oxime vs. Isoxazoles structures. J. Org. Chem., 42 ,1356-1360. doi:10.1021/jo00428a019, http://dx.doi.org/10.1021/jo00428a019

Simonis, H. S., Rosenberg, S. (1914). Uber Thiochomone , Ber,47, 1232-1237. doi:10.1002/cber.191404701197, http://dx.doi.org/10.1002/cber.191404701197

Solomons, W.E. and Doorenbos, N.J. (1974). Synthesis and antimicrobial properties of $17 \boldsymbol{\beta}$-amino-4-aza-5 $\boldsymbol{\alpha}$ -androstane and derivatives J. Pharm. Sci., 63, 19-23. doi:10.1002/jps.2600630105, http://dx.doi.org/10.1002/jps.2600630105

Spath, E., Grumber, W. (1938). Die Konstitution des Kellins (aus Ammi visnaga) (I. Mitteil. über natürliche Chromone) Ber,71, 106-113. doi:10.1002/cber.19380710118, http://dx.doi.org/10.1002/cber.19380710118

Spath, E., Grumber, W. (1941). Die Konstitution des Visnagins (aus Ammi visnaga). (II. Mitteil. über natürliche Chromone) Ber,74, 1492-1500. doi:10.1002/cber.19410740820, http://dx.doi.org/10.1002/cber.19410740820

Vargha, I., Ramonczai, J., Bathonry, J. (1949). Studies on Furan Compounds. II. Conversion of 2-Aceto-benzofuran to 2-Methyl-3-hydroxychromone J. Am. Chem. Soc., 71, 2652-2655. doi:10.1021/ja01176a014, http://dx.doi.org/10.1021/ja01176a014

Table 1. Antibacterial activities of the newly synthesized compounds:

\begin{tabular}{ccc}
\hline Sample & Inhibition zone diameter (mm $/ \mathrm{mg}$ sample) \\
\hline & $\begin{array}{c}\text { E. coli } \\
\left(\mathbf{G}^{-}\right)\end{array}$ & S. aureus \\
\hline Control DMSO & 0.0 & 0.0 \\
5a & 12 & 13 \\
5b & 13 & 11 \\
5c & 10 & 11 \\
5d & 12 & 10 \\
5e & 10 & 14 \\
5f & 11 & 11 \\
5g & 12 & 12 \\
6a & 13 & 10 \\
6b & 10 & 11 \\
6c & 13 & 14 \\
6d & 14 & 16 \\
6e & 13 & 14 \\
6f & 11 & 12 \\
6g & 11 & 13 \\
7 & 14 & 100 \\
\hline Amoxicillin & 100 & \\
\hline
\end{tabular}

1-E. coli = Escherichia coli

2-S. aureus = Staphylococcus aureus 


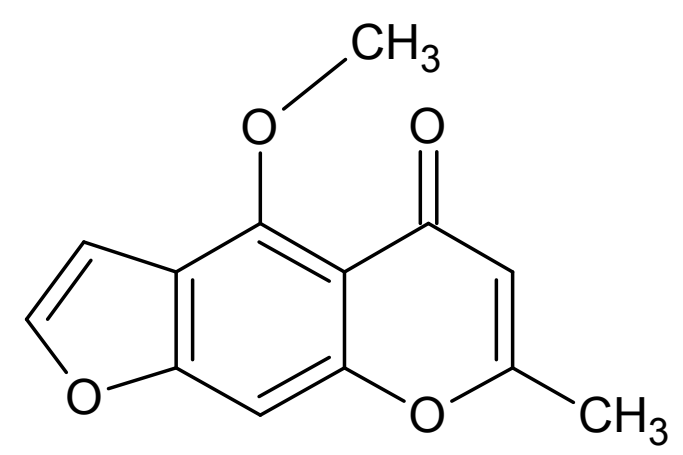

(1)

\author{
Visnagin \\ Molecular Formula $=\mathrm{C}_{12} \mathrm{H}_{8} \mathrm{O}_{5}$ \\ Formula Weight $\quad=232.18892$
}

Aralkylation reactions compounds<smiles>COc1c(C(C)=O)c(O)cc2oc(C3CCCC3C)cc12</smiles>

(1)

$$
\begin{aligned}
\text { a, } \mathrm{R} & =\mathrm{CH}_{3} \\
\text { b, } \mathrm{R} & =\mathrm{CH}_{2} \mathrm{CH}_{3} \\
\text { c, } \mathrm{R} & =\mathrm{CII}_{2} \mathrm{CII}_{2} \mathrm{CII}_{3} \\
\text { d, } \mathrm{R} & =\mathrm{CH}\left(\mathrm{CH}_{2}\right)_{2} \\
\text { e, } \mathrm{R} & =\left(\mathrm{CH}_{2}\right) \mathrm{CH}_{2} \\
\text { f, } \mathrm{R} & =\mathrm{CH}_{2}-\mathrm{CH}^{2} \mathrm{CH}_{2} \\
\text { g, } \mathrm{R} & =\mathrm{CH}_{2}-\mathrm{C}_{6} \mathrm{H}_{5}
\end{aligned}
$$<smiles>[R]Oc1cc2occc2c(OC)c1C(C)=O</smiles>

(3)

Scheme I 
Synthesis of the thiosemicarbazones and Synthesis of the 1,2,4-triazole derivatives<smiles>[R]C(C)(Cl)CC</smiles>

( 7 )<smiles>[R]Oc1cc2occc2c(OC)c1/C(C)=N/NC(N)=S</smiles>

(5)

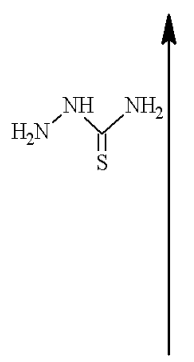<smiles>NNC(=O)NCCCCC(=O)O</smiles><smiles>CC(C)(O)Cl</smiles><smiles>COc1c(C(C)=O)c(O)cc2occc12</smiles>

(2)<smiles>[R]Oc1cc2oc(CC(N)=S)cc2c(OC)c1C1(C)NNC(=S)N1</smiles>

(6)
a, $\mathrm{R}=\mathrm{CH}_{3}$
b, $\mathrm{R}=\mathrm{CH}_{2} \mathrm{CH}_{3}$
c, $\mathrm{R}=\mathrm{CH}_{2} \mathrm{CH}_{2} \mathrm{CH}_{3}$
$\mathrm{d}, \mathrm{R}=\mathrm{CH}\left(\mathrm{CH}_{2}\right)_{2}$
$\mathrm{e}, \mathrm{R}=\left(\mathrm{CH}_{2}\right) \mathrm{CH}_{2}$
f, $\mathrm{R}=\mathrm{CH}_{2}-\mathrm{CH}=\mathrm{CH}_{2}$
g, $\mathrm{R}=\mathrm{CH}_{2}-\mathrm{C}_{6} \mathrm{H}_{5}$

\section{Scheme II}

